\title{
Aplicações da Psicologia Positiva no Desenvolvimento Infantil: Uma Revisão de Literatura
}

\author{
Patricia Cristina Ferreira ${ }^{1}$ \\ Karen Cristina Alves Lamas ${ }^{1}$ \\ ${ }^{1}$ Universidade Salgado de Oliveira, Juiz, de Fora, Minas Gerais, Brasil
}

\begin{abstract}
Resumo
O objetivo deste estudo foi realizar uma revisão integrativa da literatura brasileira sobre as aplicações da Psicologia Positiva no desenvolvimento infantil. Efetuou-se uma busca por artigos científicos nas bases de dados SciELO e PePSIC, com os termos psicologia positiva, resiliência, otimismo, habilidades sociais, bem-estar e felicidade articulados aos termos desenvolvimento infantil, crianças e infância. Foram recuperadas 202 publicações entre os anos 2000 e 2019, entre estas 72 foram analisadas. Observou-se o aumento da produção científica, com destaque para o construto habilidades sociais. Houve predomínio de pesquisas empíricas e análises quantitativas. Contudo, verificou-se poucos estudos sobre a eficácia de intervenções. Conclui-se que é importante diversificar os construtos investigados e investir em desenvolvimento e validação de instrumentos de avaliação e intervenções em diferentes contextos, que proporcionem a potencialização das qualidades humanas na infância em caráter de prevenção e promoção de saúde mental ao longo da vida.

Palavras-chave: psicologia positiva, bem-estar, crianças, infância, pesquisa científica
\end{abstract}

Applications of Positive Psychology in Child Development: A Literature Review

\begin{abstract}
This study aimed to perform an integrative review of the Brazilian literature on the applications of Positive Psychology in child development. We searched for scientific articles using the SciELO and PePSIC electronic databases, with the terms Positive Psychology, resilience, optimism, social skills, well-being, and happiness articulated to the terms child development, children, and childhood. A total of 202 publications were retrieved between 2000 and 2019, among which 72 were analyzed. We observed an increase in the scientific production on the field, especially about the social skills construct. There was a predominance of empirical research and quantitative analyses. However, there were few studies on the effectiveness of interventions. It was concluded that it is important to diversify the investigated constructs and to invest in the development and validation of evaluation instruments and interventions in different contexts that provide the enhancement of human qualities in childhood for the prevention and promotion of mental health throughout life.

Keywords: positive psychology; well-being; children; childhood; scientific research
\end{abstract}

Aplicaciones de la Psicología Positiva en el Desarrollo Infantil: Una Revisión de Literatura

\section{Resumen}

El objetivo de este estudio fue realizar una revisión integradora de la literatura brasileña sobre las aplicaciones de la Psicología Positiva en el desarrollo infantil. Se realizó una búsqueda de artículos científicos en las bases de datos SciELO y PePSIC, con los términos Psicología Positiva, resiliencia, optimismo, habilidades sociales, bienestar y felicidad, articulados con los términos desarrollo infantil, niños e infancia. Se recuperaron 202 publicaciones entre los años 2000 y 2019, entre esas 72 fueron analizadas. Se observó aumento en la producción científica, con destaque para el constructo habilidades sociales. Predominó la investigación empírica y análisis cuantitativo. Sin embargo, ha habido pocos estudios sobre eficacia de las intervenciones. Se concluye que es importante diversificar los constructos investigados e investir en desarrollo y validación de instrumentos de evaluación e intervenciones en diferentes contextos, que proporcionen mejora en las cualidades humanas en la infancia, en el campo de la prevención y promoción de salud mental a lo largo de la vida.

Palabras clave: psicología positiva; bienestar; niños; infancia; investigación científica

\section{Introdução}

Desde 1947 a Organização Mundial da Saúde define como saudável o indivíduo que possui “o completo bem-estar biológico, psicológico e social e não apenas a ausência da doença” (WHO, 1947). Partindo desse conceito amplo de saúde, Feldman e Papalia (2013) relatam que os cientistas do desenvolvimento estudam três principais domínios do eu, que são inter-relacionados: físico, cognitivo e psicossocial. Sendo que o domínio psicossocial, foco deste trabalho, abrange os aspectos personalidade, emoções e relações sociais.

No Brasil, o Estatuto da Criança e do Adolescente - ECA (Brasil, 1990) afirma que o desenvolvimento integral da criança, a partir de padrões de qualidade de vida adequados às necessidades físicas, mentais e de desenvolvimento social, é direito fundamental e 
contribui com o bem-estar do indivíduo na vida adulta. Isso indica a relevância da pesquisa no âmbito do desenvolvimento infantil como promoção de saúde no curso do desenvolvimento adulto.

Para Feldman e Papalia (2013), não há como definir objetivamente o momento em que uma criança se torna adulta, consideram o conceito de infância uma construção social, que difere de acordo com o país e sua cultura. O modelo geralmente aceito nas sociedades industriais ocidentais divide o período da infância em três fases, a primeira infância corresponde ao período de 0 a 3 anos, a segunda, de 3 a 6 anos e a terceira se inicia aos 6 e termina aos 11 anos aproximadamente, quando, inicia-se a adolescência (Feldman \& Papalia, 2013). No Brasil, o ECA, no artigo $2^{\circ}$ das disposições preliminares, considera criança a pessoa até 12 anos de idade incompletos (Brasil, 1990).

A partir disso, nota-se que a qualidade de vida na fase adulta depende de um desenvolvimento infantil saudável. De acordo com essa perspectiva, Snyder e Lopez (2009) destacam que a abordagem científica e aplicada que enfatiza a importância de descobrir e desenvolver os pontos fortes das pessoas promovendo o seu funcionamento positivo desde a infância é a Psicologia Positiva. Ainda segundo esses autores, os estudos são direcionados para a identificação, compreensão e criação de estratégias de desenvolvimento das qualidades humanas em busca de uma vida mais feliz e produtiva.

A Psicologia Positiva estuda os fundamentos psicológicos do bem-estar e da felicidade, as emoções positivas, bem como os pontos fortes e virtudes humanas (Seligman, 2011). Segundo Seligman (2011), bem-estar subjetivo corresponde ao que as pessoas sentem e pensam sobre suas vidas e ao nível de satisfação da vida percebido pelo indivíduo, um termo mais aceito cientificamente para o que as pessoas, no senso comum, chamam de felicidade. A Teoria do Bem-Estar desenvolvida por Seligman (2011) tem como base cinco elementos: emoção positiva, engajamento, sentido, relacionamentos positivos e realização. Segundo Gasparetto, Bandeira e Giacomoni (2017), o bem-estar subjetivo deve ser trabalhado ainda na infância, enquanto os traços de personalidade estão em desenvolvimento. Dessa forma, haveria a possibilidade de prevenção de repercussões negativas na vida adulta, por exemplo, para crianças que apresentam elevados níveis de neuroticismo.

Diferente da Psicologia tradicional, que historicamente, tem focado em estudar e tratar as patologias humanas (Koller \& Paludo, 2007), a Psicologia Positiva tem como foco o estudo do bem-estar, excelência e ótimo funcionamento, utilizando-se do mesmo rigor metodológico que as demais áreas da ciência psicológica (Donaldson, Dollwet, \& Rao, 2014). Como proposto por Seligman (2002) a Psicologia deveria identificar e fortalecer o que é bom, nutrir o que existe de melhor nos indivíduos, e não apenas reparar o que está errado. Consequentemente, um dos objetivos principais da Psicologia Positiva é promover o potencial e o bem-estar humano (Hutz, 2014) principalmente na infância (Seligman, Ernst, Gillham, Reivich, \& Linkins, 2009). Portanto, é necessário compreender o processo do desenvolvimento infantil partindo de uma ótica diferenciada, do fracasso, dos distúrbios, dificuldade e doenças para uma perspectiva da Psicologia Positiva que parte das competências, qualidades, sucesso e saúde (Nakano \& Siebra, 2018). Em outras palavras, sair do paradigma da doença para o da saúde (Guzzo, 2011).

Shankland e Rosset (2017) apresentaram dezesseis intervenções em Psicologia Positiva que foram testadas em contextos escolares. Eles buscaram descrever programas que pudessem ser executados por professores, integrados ao currículo existente, dispensados de burocracias administrativas, não requeriam treinamento ou materiais especiais, adaptáveis a diferentes alunos e tipos de escola e que visassem o aumento das qualidades positivas em vez de corrigir as fraquezas. As intervenções tinham como foco a promoção da atenção plena, gratidão, forças de caráter e relacionamentos positivos.

Donaldson, Dollwet e Rao (2014) concluíram, por meio de uma revisão sistemática, que tem ocorrido um aumento consistente na taxa de publicações sobre Psicologia Positiva, com destaque para os estudos empíricos. Eles identificaram 1.336 publicações entre 1999 e 2013, por meio de várias bases de dados e com artigos em língua inglesa, utilizando apenas o termo "psicologia positiva".

No contexto brasileiro, percebe-se, também, o crescente interesse dos pesquisadores por esse movimento (Koller \& Paludo, 2007) tendo como foco a infância (Dell Aglio, 2003; Gasparetto, Bandeira, \& Giacomoni, 2017; Maia \& Bortolini, 2012; Yunes, 2003). Embora nem sempre utilizem o termo, Psicologia Positiva, os estudos estão inseridos nesse paradigma (Pureza, Kuhn, Castro, \& Lisboa, 2012) e abordam temas como resiliência (Yunes, 2003), processos de coping (Dell Aglio, 2003), assertividade (Maia \& Bortolini, 2012) e bem-estar subjetivo (Gasparetto et al., 2017).

Psico-USF, Bragança Paulista, v. 25, n. 3, p. 493-505, jul./set. 2020 
Reppold, Gurgel e Schiavon (2015) e Pires, Nunes e Nunes (2015) encontraram evidências importantes, quanto ao crescente interesse de pesquisadores nacionais por estudos dos aspectos positivos do ser humano, em suas pesquisas de revisão sistemática da literatura com os temas "Pesquisas em Psicologia Positiva" e "Instrumentos baseados em Psicologia Positiva no Brasil", respectivamente, o que demonstra mudança de perspectiva na pesquisa em Psicologia.

No entanto, Reppold et al., (2015) não destacaram os estudos sobre a infância e aplicações da Psicologia Positiva nessa fase do desenvolvimento. Esses autores encontraram em maior quantidade estudos teóricos seguidos daqueles voltados para construção de instrumentos de avaliações e, por fim, intervenções. Apesar dos grandes avanços encontrados, concluem que a literatura nacional sobre o tema ainda apresenta lacunas importantes a serem sanadas, especialmente em relação à prática e à avaliação de intervenções (Reppold, Gurgel, \& Schiavon, 2015).

Diante da relevância da promoção de um desenvolvimento saudável na infância, bem como das contribuições que a Psicologia Positiva tem promovido nesse campo enquanto novo paradigma científico, questiona-se qual é o panorama atual da produção científica brasileira sobre as aplicações da Psicologia Positiva no desenvolvimento infantil. Para responder a essa questão, foi realizada uma revisão integrativa da literatura nacional sobre o tema, sem delimitação de período de tempo.

Como objetivos específicos, buscou-se: caracterizar as publicações quanto autoria, instituições, periódicos e ano de publicação; analisar os construtos que têm sido abordados nas pesquisas; verificar a prevalência de pesquisas teóricas e empíricas; analisar as características metodológicas; levantar instrumentos de avaliação utilizados; conhecer os contextos e os tipos de intervenções que possuem como alvo o público infantil no Brasil.

Espera-se que este estudo possa apresentar quais são as aplicações teóricas e práticas da Psicologia Positiva no desenvolvimento infantil que já foram empreendidas no contexto nacional. A partir do que já foi abordado e das lacunas existentes, a expectativa é que pesquisadores desenvolvam novos estudos e que os profissionais reapliquem na prática os resultados de sucesso (Zanini, Faria, \& Campos, 2018). De modo a multiplicar o número de crianças que possam obter os benefícios dessa nova visão em Psicologia, bem como possam enfrentar as adversidades da vida de forma mais saudável e adaptativa, gerando expectativas favoráveis em relação ao futuro.

\section{Método}

As revisões de literatura sistematizadas podem ser classificadas como revisão integrativa, revisão sistemática ou meta-análise. O presente trabalho caracteriza-se como uma revisão integrativa, pois tem como objetivo realizar um levantamento da literatura empírica e/ou teórica para fornecer uma compreensão mais abrangente de um determinado fenômeno, abordando conceitos e teorias, métodos de pesquisa, implicações práticas, lacunas e necessidades de futuras de pesquisas. Diferencia-se, portanto, da revisão sistemática e dos estudos de meta-análise que almejam exaustão das fontes de informação e priorizam os resultados de pesquisas empíricas, entre outras particularidades (Russel, 2005; Whittemore \& Knafl, 2005).

Para cumprir o objetivo de realizar uma revisão das publicações brasileiras sobre Psicologia Positiva e Desenvolvimento Infantil, efetuou-se uma busca a partir das bases de dados SciELO (Scientific Electronic Library Online) e PePSIC (Periódicos Eletrônicos em Psicologia). Essas fontes foram escolhidas pela relevância que possuem para a área da Psicologia no Brasil (Zoltowski, Costa, Teixeira, \& Koller, 2014). A SciELO é uma biblioteca eletrônica que abrange uma coleção selecionada de periódicos científicos brasileiros dos mais diversos campos do conhecimento. E a PePSIC é uma das mais importantes bases de dados que reúne revistas científicas de Psicologia do Brasil.

Para definir os termos de busca, recorreu-se às terminologias em Psicologia da Biblioteca Virtual em Saúde/Psicologia (BVS-Psi). Nessa plataforma foram identificados os seguintes descritores referentes à Psicologia Positiva: "resiliência", "psicologia positiva" e "otimismo". Quanto ao contexto infantil, encontraram-se as palavras: "infância", "crianças" e "desenvolvimento infantil". Devido à ideia de que a Psicologia Positiva tem como foco de estudo o "bem-estar", optou-se por incluir esse termo como fonte de busca. Foram incluídos também os descritores "habilidades sociais" e "felicidade" por serem construtos muito associados à Psicologia Positiva.

Assim, em cada base de dados, os descritores foram inseridos de modo a articular os termos da Psicologia Positiva e do Desenvolvimento Infantil utilizando o operador booleano "and", em qualquer campo/todos os índices, sem limite de tempo. Os resultados sobre o 
número de publicações recuperadas por meio dos pares de palavras em cada base são apresentados na Tabela 1. Ressalta-se que as combinações que não resultaram em recuperação de artigos nas duas bases de dados não foram expostas.

As combinações que resultaram em mais artigos foram aquelas articuladas com o termo "habilidades sociais", e as que resultaram menos, foram as combinações com o termo "psicologia positiva". Nota-se que, após a recuperação, os termos relacionados ao tema Psicologia Positiva, articulados com o termo "desenvolvimento infantil" não resultaram estudos, o que pode indicar uma lacuna na produção científica da área ou uma falha no processo de indexação dos artigos. Destaca-se, também, a relevância de terem sido incluídos os termos "habilidades sociais" e "felicidade", sendo que estes não foram indicados anteriormente pelas terminologias em Psicologia da BVS-Psi. Fato que poderia ter reduzido o número de publicações recuperadas. E ainda o pequeno número de estudos publicados que relacionam os termos "psicologia positiva" e "otimismo" ao contexto infantil, pois não foram recuperadas publicações desses descritores articulados aos termos "infância" e "desenvolvimento infantil” (Tabela 1).
Para selecionar as publicações analisadas, foram utilizados os seguintes critérios de inclusão: (1) estudos realizados no Brasil e que contenham pelo menos um autor brasileiro, (2) que investigassem construtos que fazem parte da abordagem teórica da Psicologia Positiva, (3) que tivessem como foco a infância, período entre 0 e 12 anos, (4) e que o texto completo estivesse disponível. Não houve critérios de exclusão de publicações a priori. A Figura 1 mostra, detalhadamente, o fluxograma dos processos de busca e seleção realizados.

Foram recuperados, no total, de acordo com as combinações dos termos, 233 artigos em 20 de junho de 2019 (Figura 1), dos quais foram excluídos os duplicados em cada base de dados, resultando um total de 202 estudos. Ao aplicar os critérios de inclusão, foram selecionados $75(37,13 \%)$ artigos. Dentre estes 75, 54 $(72 \%)$ publicações estavam disponíveis na base de dados SciELO e 21 (28\%) na base PePSIC. Contudo, três artigos estavam indexados, concomitantemente, nas duas bases de dados, portanto, foram excluídos, resultando em um total de 72 artigos para a análise.

Após a leitura dos textos completos, o processo de desenvolvimento deste estudo sistematizado foi caracterizar cada publicação selecionada em relação à autoria e instituição, periódicos e suas respectivas áreas, ano

Tabela 1.

Recuperação das Publicações de acordo com os Termos de Busca

\begin{tabular}{|c|c|c|c|c|}
\hline \multirow{2}{*}{ Termos de busca } & \multicolumn{2}{|c|}{ Recuperados } & \multicolumn{2}{|c|}{ Selecionados } \\
\hline & SciELO & PePSIC & SciELO & PePSIC \\
\hline Psicologia Positiva AND Crianças & 1 & 2 & 0 & 1 \\
\hline Resiliência AND Infância & 10 & 6 & 1 & 1 \\
\hline Resiliência AND Crianças & 36 & 18 & 9 & 1 \\
\hline Resiliência AND Des. Infantil & 3 & 1 & 1 & 0 \\
\hline Otimismo AND Crianças & 8 & 6 & 4 & 4 \\
\hline Bem-estar AND Infância & 1 & 1 & 0 & 1 \\
\hline Bem-estar AND Crianças & 3 & 3 & 1 & 1 \\
\hline Habilidades sociais AND Infância & 4 & 7 & 1 & 2 \\
\hline Habilidades sociais AND Crianças & 51 & 37 & 34 & 12 \\
\hline Habilidades sociais AND Des. Infantil & 3 & 1 & 3 & 0 \\
\hline Felicidade AND Infância & 5 & 3 & 2 & 1 \\
\hline Felicidade AND Crianças & 15 & 7 & 3 & 3 \\
\hline Publicações por base de dados & 141 & 92 & 59 & 27 \\
\hline Total de publicações & \multicolumn{2}{|c|}{233} & \multicolumn{2}{|c|}{86} \\
\hline Artigos analisados & & & \multicolumn{2}{|c|}{72} \\
\hline
\end{tabular}




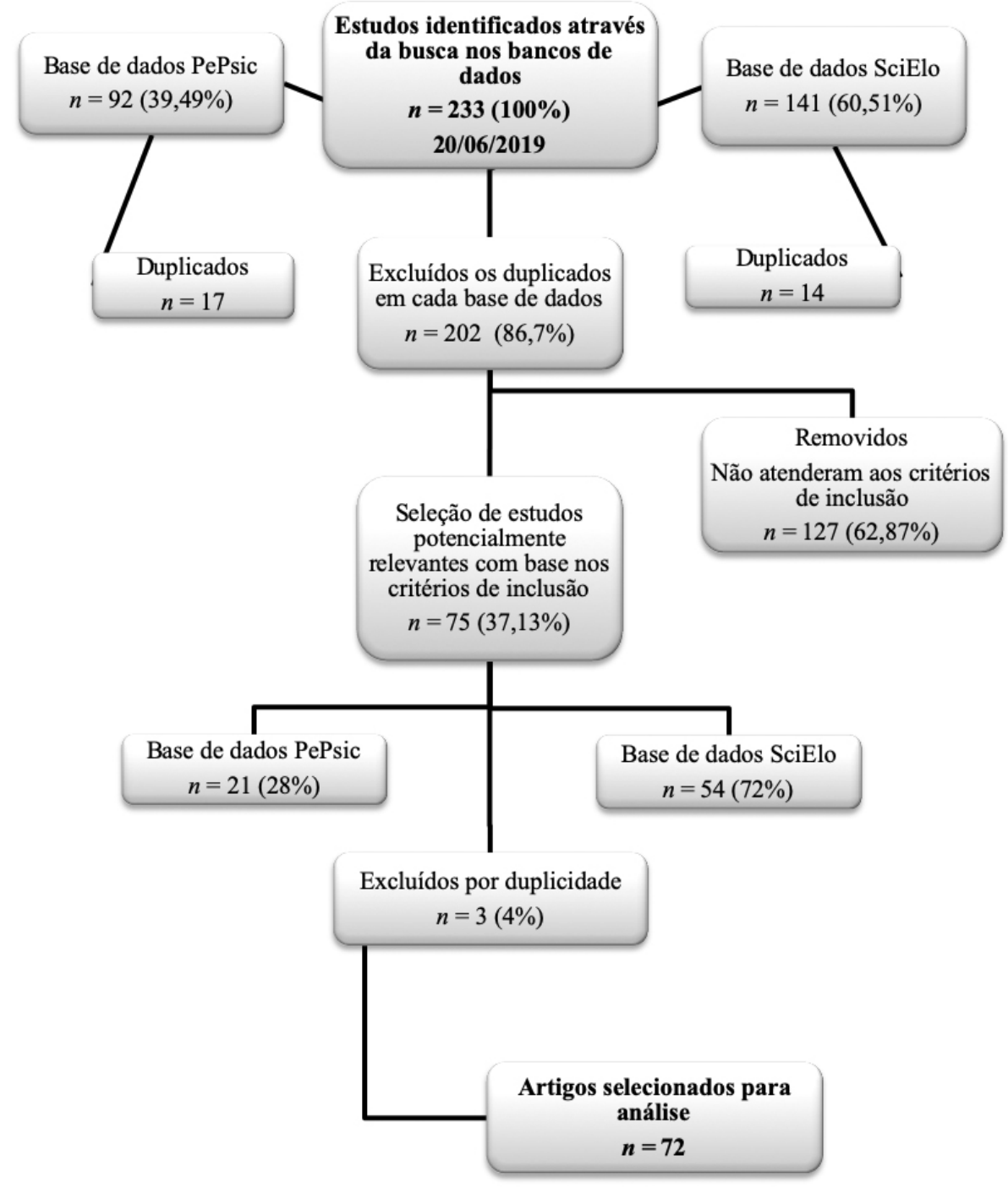

Figura 1. Fluxograma do processo de revisão sistemática.

de publicação, construtos investigados, variáveis metodológicas e características de avaliação e intervenção. Buscando a possibilidade de concluir sobre o que a literatura informa em relação às aplicações da Psicologia Positiva no contexto do desenvolvimento infantil. As informações reunidas pela investigação foram organizadas e digitadas em planilha eletrônica, criteriosamente, para garantir a qualidade e a fidedignidade dos resultados a serem publicados.

\section{Resultados e Discussão}

Os resultados e as referentes discussões são dispostos em ordem de análise e critérios de investigação, de modo a atender aos objetivos gerais e específicos.
Para referenciar as afirmações descritas, os artigos recuperados são citados em caráter de exemplificação.

Inicialmente, é importante destacar que grande parte dos artigos $(n=49 ; 68,05 \%)$ incluídos nesta revisão investiga qualidades humanas, mas não menciona o termo Psicologia Positiva. Isso demonstra que a Psicologia Positiva está sendo adotada como um paradigma ou um movimento dentro da Psicologia (Koller \& Pauldo, 2007), o que também foi identificado por Pureza, Kuhn, Castro e Lisboa (2012) em uma revisão sistemática sobre o tema.

A primeira categoria investigada foi a autoria dos estudos. A variação do número de autores foi de um a sete, sendo que a predominância foi de artigos escritos em parceria $98,06 \%(n=71)$, em sua maioria entre dois 
$(n=30 ; 41,67 \%)$ e três $(n=27 ; 37,50 \%)$ autores. Dos artigos analisados, $35(48,61 \%)$ foram escritos por autores vinculados a uma única instituição, dentre eles, seis $(8,33 \%)$ são da Universidade Federal do Rio Grande do Sul, onde fica o principal centro de pesquisa em Psicologia Positiva no Brasil (Hutz, 2014) e sete $(9,72 \%)$ da Universidade Federal de São Carlos. E, ainda, 28 artigos $(38,89 \%)$ publicados em parceria entre professores de universidades e pesquisadores, exclusivamente, bolsistas do CNPq (Conselho Nacional de Desenvolvimento Científico e Tecnológico). Dentre esses trabalhos, sete $(9,72 \%)$ foram em colaboração com universidades internacionais: três em parceria com a Universidade do Porto, de Portugal, um com a Dalhousie Universty do Canadá, um com a University of Pittsburgh e um com University of California, ambas dos Estados Unidos. No presente estudo as universidades públicas mantiveram a liderança $(n=55 ; 76,45 \%)$ em quantidade de publicações corroborando resultados já apresentados por Pires et al., (2015) em sua revisão.

Quanto aos periódicos, foram encontrados 24 títulos distribuídos em três áreas distintas, Psicologia, Educação e Medicina, o que demonstra ser um tema predominante e originário da Psicologia, mas que desperta interesse de outras áreas de conhecimento, uma vez que a primeira foi dominante em $95,83 \%(n=49)$ das revistas. O periódico que aparece com maior frequência é "Psicologia: Reflexão e Crítica" com 12 (16,67\%) publicações, seguida de "Psico-USF" com nove publicações $(12,50 \%)$ e Estudos de Psicologia (Campinas)" com seis (8,33\%). "Psicologia: Teoria e Pesquisa" e "Paidéia" também apresentaram frequência expressiva de cinco $(6,94 \%)$ artigos cada. As demais apresentaram frequência entre uma e quatro publicações. Entre as revistas citadas anteriormente, foi possível constatar a excelente qualidade científica das publicações em Psicologia Positiva, e tamanha relevância, pois as revistas com maior número de publicações possuem conceitos A1 e A2 no Qualis Periódicos.

Em relação ao ano de publicação, foram recuperados artigos entre 2000 e 2019 (Figura 2), com média de $3,78(D P=2,07)$ publicações ao ano e destaque para os anos de 2016 com oito (11,11\%) trabalhos, 2015 com sete $(9,72 \%)$ e os anos de 2011 e 2017 ambos com seis $(8,33 \%)$. Os resultados mostram que as publicações brasileiras recuperadas por este estudo, com o tema Psicologia Positiva associado ao desenvolvimento infantil, também tiveram início nos anos 2000, assim como apresentado na revisão de Pires et al., (2015) sobre os instrumentos baseados em Psicologia Positiva. Houve aumento expressivo nas publicações de estudos com crianças a partir de 2011, em que a média passou de 2,4 publicações ao ano entre 2000 e 2010 para 5,33 publicações entre 2011 e 2019, confirmando um constante crescimento da área no Brasil (Hutz, 2014).

Observa-se que a literatura nacional segue a literatura estrangeira, ainda que em menores proporções, na qual os resultados apontam um rápido crescimento em publicações relacionadas à Psicologia Positiva (Donaldson et al., 2014). Cabe lembrar que esta revisão foi feita sem recorte de tempo, assim como em outros estudos com a mesma temática (Donaldson et al., 2014; Pires, Nunes, \& Nunes, 2015; Pureza et al., 2012; Reppold et al., 2015). Importante destacar também, que o presente levantamento foi feito no mês de junho de 2019, fato que pode gerar divergência de resultados recuperados em períodos posteriores.

No que se refere aos construtos investigados, foi encontrada uma grande discrepância nos dados levantados (Tabela 2). Sobressaiu o construto "habilidades sociais" isoladamente, como objetivo principal dos estudos, e também o mesmo associado a outros, como "autoconceito", "dificuldade de aprendizagem", "emoções", "problemas de comportamento" e "expressividade facial das emoções", totalizando $61,16 \%$ (n $=44)$ dos artigos analisados. Com baixa frequência e representatividade inferior, tem-se o termo "resiliência" seguido dos construtos "felicidade", "otimismo" e "bem-estar". Tais resultados divergem daqueles encontrados por outros estudos brasileiros de revisão (Pureza et al, 2012; Pires et al., 2015), que encontraram maior representatividade de pesquisas que investigaram o construto bem-estar, porém com amostras compostas, geralmente, por participantes na idade adulta. No contexto internacional, o construto principal tem sido,

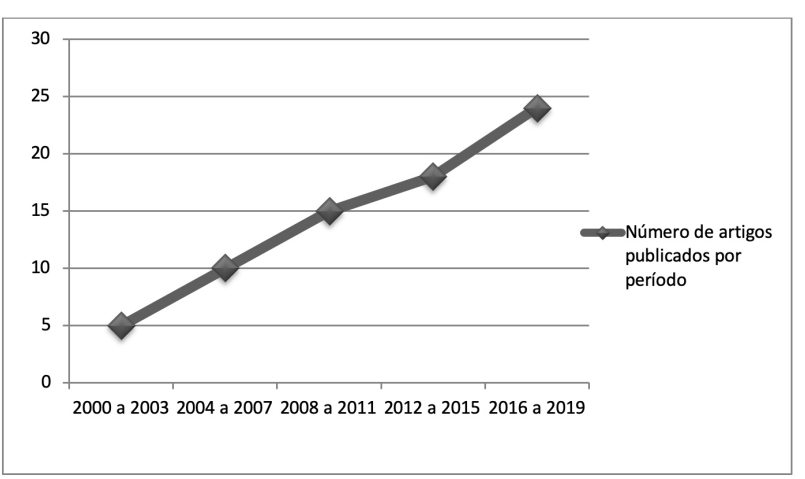

Figura 2. Publicações em Psicologia Positiva e Desenvolvimento Infantil entre 2000 e 2019. 
também, o bem-estar, seguido de forças de caráter, esperança, gratidão resiliência e crescimento, novamente, em amostras com predominância de adultos (Donaldson et al., 2014).

Salienta-se que o construto felicidade, que era raramente utilizado em pesquisas científicas pelo caráter subjetivo de seu conceito (Snyder \& Lopez, 2009), já aparece de forma notável nesta revisão, o que mostra o interesse da ciência por esse tipo de variável e pelos aspectos positivos na infância. No entanto, não foram recuperados estudos nacionais que abordassem construtos como educação positiva, atenção plena/ mindfulness, gratidão e forças, assim como aparecem na literatura estrangeira (Seligman et al., 2009; Shankland \& Rosset, 2017). Não com os termos de busca utilizados, dentre eles os sugeridos pela BVS-Psi. Embora não tenha sido objetivo deste estudo fazer uma comparação entre os contextos nacional e internacional, é possível notar pela revisão sistemática de Donaldson et al., (2014) que a literatura nacional ainda é pequena diante da quantidade de publicações e diversidade dos temas tratados na literatura estrangeira.

O resultado da análise referente às variáveis metodológicas demonstrou que pesquisas empíricas foram as mais frequentes, com a constância de 59 artigos dentre os 72 analisados, o que corresponde a $81,94 \%$ das publicações selecionadas. Dentre os 59 estudos empíricos, 54 $(91,53 \%)$ são pesquisas de levantamento/transversais, dois $(3,39 \%)$ possuem delineamento longitudinal e dois $(3,39 \%)$ são quase experimentais. Foi predominante o método quantitativo com $81,36 \%(n=48)$ seguido do método qualitativo com $13,56 \%(n=8)$ e do método

Tabela 2.

Principais Construtos Investigados pelos Artigos Analisados

\begin{tabular}{lcc}
\hline \multicolumn{1}{c}{ Construtos investigados } & $n$ & $\%$ \\
\hline Habilidades Sociais & 37 & 51,43 \\
Habilidades Sociais + outros & 7 & 9,73 \\
construtos & & \\
Resiliência & 11 & 15,29 \\
Felicidade & 03 & 4,17 \\
Felicidade + Bem-estar subjetivo & 01 & 1,39 \\
Otimismo & 03 & 4,17 \\
Otimismo + outros construtos & 02 & 2,78 \\
Outros construtos & 8 & 11,12 \\
Total & 72 & 100 \\
\hline
\end{tabular}

misto em 5,08\% $(n=3)$ dos artigos analisados. Verifica-se que o desenvolvimento da pesquisa brasileira segue o internacional, mostrando um aumento consistente na taxa de publicação, sendo a maior parte de estudos empíricos, quantitativos e transversais (Donaldson et al., 2014), embora ainda não seja na mesma proporção (Pires et al., 2015; Pureza et al., 2012; Reppold et al., 2015). Os outros $13(18,06 \%)$ artigos analisados reportam relatos de experiência ( $n=3,4,17 \%)$, que abordam os construtos assertividade, habilidades sociais, e emoções; e estudos teóricos $(n=10,13,89 \%)$ sobre resiliência ( $n$ $=7,9,72 \%)$, habilidades sociais $(n=2,2,78 \%)$ e design emocional $(n=1,1,39 \%)$.

A seguir são apresentados e discutidos os resultados especificamente relacionados aos estudos empíricos. Ao analisar as características das amostras, salienta-se que nem todos os estudos apresentaram a média de idade dos participantes, mas indicaram a amplitude. Assim, foi necessário e possível reclassificar a idade dos componentes de acordo com os períodos do ciclo do desenvolvimento humano indicados por Papalia e Feldman (2013) e descritos anteriormente na introdução deste texto.

Os resultados indicaram predominância de estudos na Terceira Infância, com $69,49 \%(n=41)$ dos trabalhos, e $22,03 \%(n=13)$ com amostras de participantes classificados entre a Segunda e a Terceira Infância. Observa-se, então, a falta de pesquisas empíricas com foco em crianças incluídas na categoria Primeira Infância, entre 0 e 3 anos, que na presente avaliação representam apenas 1,69\%, um artigo dentre os selecionados. A carência de pesquisas nesta fase levanta algumas hipóteses, como, por exemplo, maior dificuldade de acesso a esse público, pois nem todas as crianças nessa faixa etária frequentam instituições de ensino, ou ainda, a escassez de instrumentos desenvolvidos para avaliações e intervenções validados e adequados para crianças (Pureza et al., 2012). Pires et al., (2015) verificaram, também, poucos estudos sobre instrumentos para crianças do ensino fundamental, aquelas que correspondem à categoria Segunda e Terceira Infância, e não recuperaram estudos com amostras de crianças na Primeira Infância. Donaldson et al., (2014) verificaram, também, que a menor parte dos estudos analisados tinha como foco crianças ou adolescentes.

Em relação às escolas, a maior parte dos estudos usou amostras com alunos do ensino fundamental ( $n$ $=42 ; 71,19 \%$ ), na sua maioria de instituições públicas $(n=33 ; 55,93 \%)$, apenas $15(25,41 \%)$ estudos utilizaram amostras de instituições públicas e privadas 
juntas, e 15,25\% ( $n=9)$ dos trabalhos não disponibilizaram essa informação. Destaca-se que apenas dois estudos $(3,39 \%)$ tiveram amostras compostas somente por alunos de instituição privada, demonstrando que instituições públicas tendem a ser alvo das investigações, possivelmente, devido ao caráter social da pesquisa científica.

Segundo Cintra e Guerra (2017), as instituições educacionais são locais privilegiados para a aplicação da Psicologia Positiva, tendo em vista sua importância para o desenvolvimento de indivíduos e da sociedade. As práticas podem possibilitar a promoção de bem-estar e florescimento, não só dos alunos, mas de todos que participam da vida escolar. Nesse mesmo sentido, Reppold, Gurgel e Almeida (2018) ressaltam a importância do trabalho do psicólogo escolar no desenvolvimento de programas que favoreçam aspectos positivos, como socialização, otimismo e bem-estar subjetivo e que qualifiquem o desenvolvimento emocional, cognitivo e social dos discentes, envolvendo pais e professores em suas interações.

No que diz respeito aos instrumentos de avaliação, o utilizado com maior frequência $(n=21 ; 35,49 \%)$ foi o inventário para crianças de ensino fundamental, o Sistema de Avaliação de Habilidades Sociais (Social Skills Rating System - SSRS), adaptado para o Brasil por Bandeira, Del Prette, Del Prette e Magalhães (2009), o que está em consonância com o resultado dos construtos avaliados. Esse levantamento atual sugere a carência de instrumentos para avaliar outras dimensões do referido construto, assim como já foi indicado por Bandeira et al., (2009) e por Bolsoni-Silva, Marturano e Loureiro (2011).

Em três estudos, o SSRS-BR foi associado ao Teste de Desempenho Escolar - TDE, avaliando e comprovando a relação entre habilidades sociais, comportamento e desempenho escolar (Cia \& Barham, 2009; Elias \& Amaral, 2016; Feitosa, Del Prette, Del Prette, \& Loureiro, 2011; Seligman et. al 2009). Tal relação foi contemplada também por Nakano e Siebra (2018) sobre a importância das competências socioemocionais no contexto escolar. As autoras apontam a influência positiva de atividades que desenvolvam habilidades sociais e emocionais no sucesso acadêmico, comportamental e na determinação do bem-estar ao longo da vida.

Outro instrumento que se destacou, e que também avalia aspectos positivos, foi a Escala Multidimensional de Satisfação de Vida para Crianças (EMSVC) desenvolvida para o contexto brasileiro por Giacomoni e
Hutz (2008), foi citada em três estudos que correlacionaram o bem-estar subjetivo a outros construtos, como otimismo (Bandeira, Natividade, \& Giacomoni, 2015) habilidades sociais e dotação (Freitas, Del Prette, \& Del Prette, 2017a; Freitas, Del Prette, \& Del Prette, 2017b). Nos três casos os resultados da EMSVC foram correlacionados aos da Escala de Afetos positivos e Negativos para crianças, desenvolvida pelos mesmos autores brasileiros (Giacomoni \& Hutz, 2006). A combinação das duas escalas, citadas anteriormente, é geralmente utilizada em pesquisas que tem entre os objetivos, o de mensurar o bem-estar subjetivo infantil (Giacomoni \& Hutz, 2006; 2008).

Em menor frequência, foram aplicados instrumentos que mensuram e avaliam o otimismo, como o Revised Life Orientation Test (LOT-R) adaptado para o Brasil por Bastianello, Pacico e Hutz (2014), Youth Life Orientationtest (YLOT) e Tarefas Preditoras de Otimismo em Crianças (TAPOC) que avalia o otimismo em crianças pequenas, de quatro a oito anos (Bandeira, Giacomoni, \& Hutz, 2015). Ao todo foram identificados nove instrumentos de avaliação de construtos relacionados aos aspectos positivos das crianças, o que revela a discrepância em relação aos resultados encontrados por Pires et al., (2015) em sua revisão sem o recorte da fase de desenvolvimento.

Nota-se que questionários e entrevistas, também foram utilizados em grande parte $(n=28 ; 47,32 \%)$ das publicações analisadas. Dentre eles, o Questionário de Estilo de Atribuição Comportamental de Crianças (Children's Attributional Style Questionnaire - CASQ) que avalia o otimismo na criança, foi instrumento de duas pesquisas, sendo que uma delas examina suas evidências de validade e a outra o construto otimismo, ambas associaram o CASQ com escalas de Exigência e Responsividade que analisam estilos parentais (Weber, Brandenburg, \& Viezzer, 2003; Weber, Prado, Brandenburg, \& Viezzer, 2003). O Questionário de Respostas Socialmente Habilidosas - QRSH aparece em oito artigos (p. ex., Bolsoni-Silva \& Loureiro, 2018; Bolsoni-Silva \& Loureiro, 2019; Mariano \& Silva, 2016; Silva, Mariano, Loureiro, \& Bonaccorsi, 2013), esse instrumento é utilizado para medir os níveis de habilidades sociais de crianças pela perspectiva de pais e professores. Não menos significativas, as entrevistas variam entre estruturadas e semiestruturadas, utilizadas pelos autores para levantar dados sobre construtos e comportamentos dos participantes.

Quanto às estratégias de intervenção, somente 13,56\% $(n=8)$ apresentaram em seu método a aplicação

Psico-USF, Bragança Paulista, v. 25, n. 3, p. 493-505, jul./set. 2020 
de alguma prática. Considerando a variedade de temas expostos por Shankland e Rosset (2017), destaca-se a atuação dos pesquisadores brasileiros em utilizar programas de desenvolvimento que visem aos relacionamentos positivos. Todos $(100 \%)$ os oito estudos usam projetos e programas para promoção de habilidades sociais (p. ex., Batista \& Marturano, 2015; Elias \& Amaral, 2016; Ferreira \& Munster, 2017; Paixão \& Alvarenga, 2019; Souza, Soares, \& Freitas, 2018). Os autores confirmaram a eficácia da aplicação dos programas e descreveram a melhoria significativa no nível de habilidades sociais, no autoconceito e no desempenho escolar dos participantes, promovendo relevantes contribuições à literatura e à prática profissional da Psicologia. São necessários mais experimentos que busquem a promoção de outros construtos, como gratidão, atenção plena e forças de caráter, por exemplo. Há alguns programas que estão sendo adaptados e testados em vários países (mais informações em Shankland \& Rosset, 2017) e poderiam ser implementados no Brasil, após sua adaptação transcultural.

Vale destacar que um (12,50\%) estudo testou a eficácia de uma intervenção para melhoria da expressão facial das emoções e do repertório de habilidades sociais, o "Programa para desenvolvimento da expressão facial de emoções" (Ferreira \& Del Prette, 2013). Esse construto tem grande importância para a Psicologia Positiva, pois compõe o primeiro elemento da Teoria do Bem-Estar de Seligman (2011), a emoção positiva. Os autores encontraram resultados relevantes na melhoria da expressão facial das emoções e nos relacionamentos, que foram, em geral, mantidos ou aperfeiçoadas ao longo do tempo e para além do contexto de intervenção.

Nas intervenções citadas nos parágrafos anteriores, os pesquisadores utilizaram em sua maioria $(n$ $=5 ; 62,50 \%$ a escola como ambiente para aplicação. Isso faz sentido devido à facilidade de acesso a crianças (Pires et al., 2015). Mas também é uma estratégia fundamentada teoricamente, na qual os autores tomam como base a possibilidade de educar as crianças e os jovens no contexto escolar, sob a perspectiva da Educação Positiva, desenvolvida por Seligman (2004; 2011). Essa abordagem propõe o ensino do bem-estar na escola, com programas de desenvolvimento positivo que têm como foco a promoção de resiliência e outras habilidades humanas (Cintra \& Guerra, 2017; Pureza et al., 2012; Seligman et al., 2009). Em âmbito internacional, já foi constatado que intervenções na perspectiva da Psicologia Positiva implementadas em salas de aula produziram resultados promissores, não apenas em termos de bem-estar dos alunos, mas também em termos de realização acadêmica, clima escolar e bem-estar dos professores (Shankland \& Rosset, 2017).

Embora o crescimento do interesse das pesquisas nacionais em aspectos positivos foi notável nesta pesquisa, o que se percebe ainda, em comparação com os estudos estrangeiros, é um baixo número de pesquisas interessadas na compreensão da qualidade de vida infantil, nas questões positivas da infância (Giacomoni, Souza, \& Hutz, 2014; Pureza et al., 2012) e uma carência de teorias consolidadas e instrumentos precisos e validados para sua avaliação (Campos, 2018).

\section{Considerações Finais}

Em observância ao objetivo principal deste estudo - fazer uma revisão integrativa da literatura brasileira sobre as aplicações da Psicologia Positiva no período de desenvolvimento infantil - verificou-se ainda um incipiente estágio quanto ao número de pesquisas e à variedade de instrumentos e de intervenções direcionadas a crianças, principalmente ao comparar com a literatura internacional (Donaldson et al., 2014; Shankland \& Rosset, 2017). Felizmente, notou-se relevante crescimento nos últimos anos, o que demonstra um aumento do interesse de pesquisadores por essa área da Psicologia.

Os resultados demonstraram a predominância de pesquisas empíricas com métodos quantitativos, que em sua maioria investigam o construto habilidades sociais em crianças entre 3 e 12 anos. Verificou-se, portanto, a escassez de estudos que utilizem métodos qualitativos, que trabalhem outros construtos e que tenham como foco a primeira infância. Tais estudos são relevantes para fundamentar a necessidade de novas técnicas de avaliação de características positivas (Viapiana, Bandeira, \& Giacomoni, 2016) e intervenções direcionadas para essa população. Importante destacar as pesquisas que avaliam programas de habilidades sociais que buscam a promoção de relacionamentos positivos, com resultados de validade expressivos atribuídos às intervenções. Assim comprovam sua eficácia e estimulam a criação de novos programas dentro do campo científico.

Entretanto, observaram-se lacunas importantes referentes a estudos com intervenções que visem promover desenvolvimento de bem-estar subjetivo, que é o tema central da Psicologia Positiva de Seligman (2011). Outros construtos também podem ser objeto de estudo para futuras pesquisas em Psicologia Positiva, como 
educação positiva, atenção plena/mindfulness, gratidão e forças de caráter (Seligman et al., 2009; Shankiland \& Rosset, 2017), que podem ser investigados em diversos contextos, como escolas, ambiente familiar e comunidade. O intuito é possibilitar intervenções de qualidade e apoio social, promovendo desenvolvimento positivo, indivíduos mais fortalecidos, resilientes e psicossocialmente saudáveis (Zanini et al., 2018).

Quanto às limitações, entende-se que devido à variedade de construtos abordados pela Psicologia Positiva, embora tenha havido um esforço para abranger os termos mais relevantes, alguns artigos podem não ter sido recuperados por meio das palavras utilizadas para a busca. Por exemplo, o trabalho de Cintra e Guerra (2017), que enfatizou a educação emocional positiva, e o artigo de Gasparetto et al., (2017), que avaliou a relação entre bem-estar subjetivo e traços de personalidade, não foram recuperados por meio das buscas empreendidas aqui. Possivelmente, esses artigos não foram indexados por meio de descritores que caracterizassem a fase de desenvolvimento humano que estavam abordando. Sugere-se que autores e editores fiquem atentos à caracterização, não apenas do construto abordado, mas também da população-alvo envolvida no estudo ao indexar os manuscritos, de forma a ampliar as possibilidades de recuperação e visibilidade do texto.

Ressalta-se que não foram encontradas outras revisões nacionais que investigassem aplicações da Psicologia Positiva exclusivamente no desenvolvimento infantil, sendo essa a maior contribuição do presente estudo. Uma vez que a proposta da Psicologia Positiva é o foco na construção das forças do sujeito para tratar e prevenir as desordens psicológicas (Seligman, 2002), sugere-se mais pesquisas com intervenções na fase inicial da vida em caráter de prevenção e promoção de saúde mental na vida adulta. É importante destacar a relevância de estudos com a temática tanto em contextos clínicos quanto sociais, de modo que os psicólogos conheçam a aplicabilidade da Psicologia Positiva para potencializar as qualidades humanas, com o equilíbrio entre o positivo e o negativo, promovendo uma visão mais completa do ser humano (Snyder \& Lopes, 2009).

\section{Referências}

Bandeira, M., Del Prette, Z. A. P., Del Prette, A., \& Magalhães, T. (2009). Validação das escalas de habilidades sociais, comportamentos problemáticos e competência acadêmica (SSRS-BR) para o Ensino
Fundamental. Psicologia: teoria e pesquisa, 25(2), 271282.doi: 10.1590/S0102-37722009000200016

Bandeira, C. M., Giacomoni, C. H., \& Hutz, C. S. (2015). Tarefas preditoras de otimismo em crianças (TAPOC): Construção e evidências de validade. Avaliação Psicológica: Interamerican Journal of Psychological Assessment, 14(2), 8. doi: 10.15689/ ap.2015.1402.04

Bandeira, C. M., Natividade, J. C., \& Giacomoni, C. H. (2015). As relações de otimismo e bem-estar subjetivo entre pais e filhos. Psico-USF, 20(2), 249-257. doi: 10.1590/1413-82712015200206

Batista, S. V., \& Marturano, E. M. (2015). Intervenção para promover habilidades sociais e reduzir problemas de comportamento de crianças em um núcleo social. Pesquisas e Práticas Psicossociais, 10(2), 313-326. Recuperado de http://pepsic.bvsalud. org/pdf/ppp/v10n2/09.pdf

Bastianello, M. R., \& Pacico, J. C. (2014). Otimismo. Em C. S. Hutz (Ed.), Avaliação em psicologia positiva (pp. 49-55). Artmed: Porto Alegre, Brasil.

Bolsoni-Silva, A. T., \& Loureiro, S. R. (2018). Predictors of social skills and behavioral problems in children. Arquivos Brasileiros de Psicologia, 70(1), 86-97. Recuperado de http://pepsic. bvsalud.org/scielo.php?script=sci_arttext\&pi$\mathrm{d}=$ S1809-52672018000100007\&lng=pt\&tlng=en

Bolsoni-Silva, A. T., \& Loureiro, S. R. (2019). Práticas Parentais: Conjugalidade, depressão materna, comportamento das crianças e variáveis demográficas. Psico-USF, 24(1), 69-83. doi: 10.1590/1413-82712019240106

Bolsoni-Silva, A. T., Marturano, E. M., \& Loureiro, S. R. (2011). Estudos de Confiabilidade e Validade do Questionário de Respostas Socialmente Habilidosas Versão para Pais - QRSH-Pais. Psicologia: Reflexão e Crítica, 24(2), 1-9. doi:10.1590/ S0102-79722011000200003

Brasil. (1990). Estatuto da Criança e do Adolescente. Lei Federal no 8069 de 13/07/1990. Recuperado de http://www.planalto.gov.br/ccivil_03/leis/L8069. htm

Campos, C. R. (2018). Qualidade de vida infantil e sua influência no contexto escolar. Em Nakano, T. C (Eds.), Psicologia Positiva Aplicada à Educaşão (Ed. 1). (pp. 19-40) São Paulo: Vetor.

Psico-USF, Bragança Paulista, v. 25, n. 3, p. 493-505, jul./set. 2020 
Cia, F., \& Barham, E. J. (2009). Repertório de habilidades sociais, problemas de comportamento, autoconceito e desempenho acadêmico de crianças no início da escolarização. Estudos de Psicologia, 26(1), 45-55. doi: 10.1590/S0103-166X2009000100005

Cintra, C. L., \& Guerra. V. M. (2017). Educação Positiva: A aplicação da Psicologia Positiva a instituições educacionais. PsicologiaEscolareEducacional,21(3),505514. doi: 10.1590/2175-3539/2017/021311191

Dell Aglio, D. D. (2003). O processo de coping em crianças e adolescentes: Adaptação e desenvolvimento. Temas em Psicologia da SBP, 11(1) 38-45. Recuperado de http://pepsic.bvsalud.org/pdf/ tp/v11n1/v11n1a05.pdf

Donaldson, S. I., Dollwet, M., \& Rao, M. A. (2014). Happiness, excellence, and optimal human functioning revisited: Examining the peer-reviewed literature linked to positive psychology. The Journal of Positive Psychology, 10(3), 185-195. doi: 10.1080/17439760.2014.943801

Elias, L. C. S., \& Amaral, V. A. (2016). Habilidades sociais, comportamentos e desempenho acadêmico em escolares antes e após intervenção. Psico-USF, 21(1), 49-61. doi: 10.1590/1413-82712016210105

Feitosa, F. B., Del Prette, A., Del Prette, Z. A. P., \& Loureiro, S. R. (2011). Explorando relações entre o comportamento social e o desempenho acadêmico em crianças. Estudo e pesquisas em Psicologia, 11(2) 442-455. Recuperado de http://pepsic.bvsalud. org/pdf/epp/v11n2/v11n2a05.pdf

Feldman, R. D., \& Papalia, D. E. (2013). O estudo do desenvolvimento humano. Em R. D. Feldman \& D. E. Papalia (Ed. 12), Desenvolvimento Humano. (pp. 34-53) Porto Alegre: Artmed.

Ferreira, B. C., \& Del Prette, Z. A. P. (2013). Programa de expressividade facial de emoções e habilidades sociais de crianças deficientes visuais e videntes. Psicologia: Reflexão e Crítica, 26(2), 327-338. doi: 10.1590/S0102-79722013000200013

Ferreira, E. F., \& Munster, M. A. van. (2017). Avaliação das Habilidades Sociais de Crianças com Deficiência Intelectual sob a Perspectiva dos Professores. Revista Brasileira de Educação Especial, 23(1), $97-$ 110. doi: 10.1590/s1413-65382317000100008

Freitas, M. L. P. F., Del Prette, A., \& Del Prette, Z. A. P. (2017a). Habilidades sociais e bem-estar subjetivo de crianças dotadas e talentosas. Psico-Usf, 22(1), 1-12. doi: 10.1590/1413-82712017220101

Freitas, M. L. P. F., Del Prette, A., \& Del Prette, Z. A. P. (2017b). Dotação, habilidades sociais e bem-estar subjetivo. Arquivos Brasileiros de Psicologia, 69(3), 133-148. Recuperado de http://pepsic. bvsalud.org/scielo.php?script $=$ sci_arttext\&pi$\mathrm{d}=$ S1809-52672017000300010\&lng=pt\&tlng=pt

Gasparetto, L. G., Bandeira, C., \& Giacomoni, C. H. (2017). Bem-estar subjetivo e traços de personalidade em crianças: Uma relação possível? Temas em Psicologia, 25(2), 447-457. doi: 10.9788/ TP2017.2-03

Giacomoni, C. H., \& Hutz, C. S. (2006). Escala de afeto positivo e negativo para crianças: Estudos de construção e validação. Psicologia Escolar e Educacional, 10(2), 235-245. Recuperado de http://pepsic. bvsalud.org/scielo.php?script $=$ sci_arttext\&pi$\mathrm{d}=$ S1413-85572006000200007\&lng=pt\&tlng $=\mathrm{pt}$

Giacomoni, C. H, \& Hutz, C. S. (2008). Escala multidimensional de satisfação de vida para crianças: Estudos de construção e validação. Estudos de Psicologia Campinas, 25(1), 23-35. doi: 10.1590/ S0103-166X2008000100003

Giacomoni, C. H., de Souza, L. K., \& Hutz, C. S. (2014). O conceito de felicidade em crianças. Psico-USF, 19(1), 143-153. doi: 10.1590/ S1413-82712014000100014

Guzzo, R. S. L. (2011). Saúde psicológica, sucesso escolar e eficácia da escola. Em Z. A. P. Del Prette (Ed.). Psicologia escolar e educacional: Saúde e qualidade de vida - explorando fronteiras. (pp. 19-36) Campinas: Alínea.

Hutz, C. S. (Ed.). (2014). Avaliação em Psicologia Positiva. Porto Alegre: Artmed.

Koller, S. H., \& Paludo, S. S. (2007). Psicologia Positiva: Uma nova abordagem para antigas questões. Paidéia, 17(36), 9-20. doi: 10.1590/ S0103-863X2007000100002

Maia, D. S., \& Bortolini, M. (2012). O desenvolvimento da habilidade de assertividade e a convivência na escola: Relato de experiência. Psicologia em revista, 8(3), 373388. doi: 10.5752/P.1678-9563.2012v18n3p373

Mariano, M., \& Silva, A. T. B. (2016). Comparações entre práticas educativas de professores, habilidades sociais e problemas de comportamento de alunos. 
Estudos e Pesquisas em Psicologia, 16(1), 140-160. Recuperado de http://pepsic.bvsalud.org/pdf/epp/ v16n1/v16n1a09.pdf

Nakano, T. C., \& Siebra, M. V. S. (2018). Competências socioemocionais no contexto educacional: Importância e usos. Em Nakano, T. C (Org.), Psicologia Positiva Aplicada à Educação (Ed. 1). (pp. 63-80) São Paulo: Vetor.

Paixão, C., \& Alvarenga, P. (2019). Effects of an Intervention on Social Competence of Children Surviving Brain Tumors. Paidéia (Ribeirão Preto), 29. doi: 10.1590/1982-4327e2914

Pires, J. G., Nunes, C. H. S. S., \& Nunes, M. F. O. (2015). Instrumentos baseados em psicologia positiva no Brasil: Uma revisão sistemática. Psico-USF, 20(2), 287-295. doi: 10.1590/1413-82712015200209

Pureza, J. R., Kuhn, C. H. C., Castro, E. K., \& Lisboa, C. S. M. (2012). Psicologia positiva no Brasil: Uma revisão sistemática da literatura. Revista Brasileira de Terapia Cognitiva, 8(2), 109-117. doi: 10.5935/1808-5687.20120016

Reppold, C. T. Gurgel, L. G., \& Almeida, L. S. (2018). Intervenções de psicologia positiva no contexto da psicologia escolar. Em Nakano, T. C (Eds.), Psicologia Positiva Aplicada à Educação (Ed. 1). (pp. 7-18) São Paulo: Vetor.

Reppold, C. T., Gurgel, L. G., \& Schiavon, C. C. (2015). Pesquisas em Psicologia Positiva: Uma revisão sistemática da literatura. Psico-USF, 20(2), 275-285. doi: 10.1590/1413-82712015200208

Russell, C. L. (2005). An overview of the integrative research review. Progress in transplantation, 15(1), 8-13. Recuperado de https://www.researchgate. net/publication/7898657_An_overview_of_the_ integrative_research_review

Seligman, M. E. P. (2002). Positive psychology, positive prevention and positive therapy. Em C. R. Snyder \& S. L. Lopez. (Eds.) Handbook of positive psychology. New York: Oxford University Press.

Seligman, M. E. P. (2004). Felicidade Autêntica. Rio de Janeiro: Objetiva.

Seligman, M. E. P. (2011). Florescer: Uma nova e visionária interpretação da felicidade e do bem-estar. Rio de Janeiro: Objetiva.
Seligman, M. E. P., Ernst, R. M., Gillham, J., Reivich, K. \& Linkins, M. (2009) Positive education: Positive psychology and classroom interventions. Oxford Review of Education, 35(3), 293-311. doi: 10.1080/03054980902934563

Shankland, R. \& Rosset, E. (2017). Review of brief school-based positive psychological interventions: A taster for teachers and educators. Educational Psychology Review, 29(2), 363-392. doi: 10.1007/ s10648-016-9357-3

Shankland, R., \& Rosset, E. (2017). Review of Brief School-Based Positive Psychological Interventions: a Taster for Teachers and Educators. Educational Psychology Review, 29, 363-392. doi: 10.1007/ s10648-016-9357-3

Silva, A. T. B., Mariano M. L., Loureiro S. R., \& Bonaccorsi, C. (2013). Contexto Escolar: Práticas educativas do professor, comportamento e habilidades sociais infantis. Revista Semestral da Associação Brasileira de Psicologia Escolar e Educacional, 17(2), 259-269.doi: 10.1590/S1413-85572013000200008

Souza, M. S. D., Soares, A. B., \& Freitas, C. P. P. D. (2018). Efeitos de um treinamento de habilidades sociais no comportamento e desempenho acadêmico. Avaliação Psicológica, 17(4), 417-427. doi: 10.15689/ap.2018.1704.2.02

Snyder, C. R., \& Lopez, S. J. (2009). Psicologia Positiva: Uma abordagem cientifica e prática das qualidades humanas (R. C. Costa, Trad.). Porto Alegre: Artmed.

Viapiana, V. F., Bandeira, C. M., \& Giacomoni, C. H. (2016). Bem-estar subjetivo infantil: Avaliação por meio do desenho da figura humana. Avaliação Psicológica, 15(1), 49-59. doi: 10.15689/ap.2016.1501.06

Weber, L. N. D., Brandenburg, O. J., \& Viezzer, A. P. (2003). A relação entre estilo parental e o otimismo da criança. Psico-USF, 8(1), 71-79. doi: 10.1590/ S1413-82712003000100010

Weber, L. N. D., Prado, M. P., Brandenburg, O. J., \& Viezzer, A. P. (2003) Avaliação da validade do questionário de Estilo de Atribuição para Crianças (CASQ). Psicologia Escolar e Educacional, 7(2), 161170. Recuperado de http://pepsic.bvsalud.org/ pdf/pee/v7n2/n2a06.pdf

Whittemore, R., \& Knafl, K. (2005). The integrative review: Updated methodology. 
Journal of Advanced Nursing, 52(5), 546-553. doi: 10.1111/j.1365-2648.2005.03621.x

World Health Organization. (1947). World Health Organization Constitution. Genebra: World Health Organization. Recuperado de http://www.who. int/en/

Yunes, M. A. A. (2003). Psicologia Positiva e Resiliência: $O$ foco no indivíduo e na família. Psicologia em Estudo, 8(esp.), 75-84. doi: 10.1590/ S1413-73722003000300010

Zanini, D. S., Faria, M. R. G. V., \& Campos, D. C. (2018). Fatores psicossociais da violência e desempenho escolar de crianças e adolescentes: Contribuições da Psicologia Positiva. Em Nakano, T. C (Eds.), Psicologia Positiva Aplicada à Educação (Ed. 1). (pp. 161-177) São Paulo: Vetor.

Zoltowski, A. P. C., Costa, A. B., Teixeira, M. A. P., \& Koller, S. H. (2014). Qualidade metodológica das revisões sistemáticas em periódicos de psicologia brasileiros. Psicologia: teoria e Pesquisa, 30(1), 97-104. doi: 10.1590/S0102-37722014000100012

Recebido em: 28/10/18

Reformulado em: 28/07/2019

Aprovado em: 28/08/2019

Sobre as autoras:

Patricia Cristina Ferreira é psicóloga, pós-graduada em Terapia Cognitivo-Comportamental pela Universidade Salgado de Oliveira - UNIVERSO/Juiz de Fora.

ORCID: https://orcid.org/0000-0001-9040-2908

E-mail: patriciaferreirapsico@outlook.com

Karen Cristina Alves Lamas é psicóloga, doutora em Psicologia pela Universidade São Francisco e docente do curso de graduação em Psicologia da Universidade Salgado de Oliveira - UNIVERSO/Juiz de Fora.

ORCID: https://orcid.org/0000-0003-1029-0008

E-mail:karen_lammas@yahoo.com.br

Contato com as autoras:

Universidade Salgado de Oliveira - UNIVERSO

Av. dos Andradas, 731, Morro da Glória

Juiz de Fora-MG, Brasil

CEP: 36036-000

Telefone: (32) 3311-9917 